\title{
Potential Benefits of Using Commercial Simulators To Test Equipment Control Systems
}

\author{
Francis Ruppel and Wes Wysor \\ Oak Ridge National Laboratory*
}

P.O. Box 2008

Oak Ridge, TN 37831-6003

\section{Prepared for the}

SEMI/IEEE Advanced Semiconductor Manufacturing Conference \& Workshop (ASMC)

September 10-12, 1997

Cambridge, $\mathrm{MA}$

The submitted manuscript has been authored by a contractor of the U.S. Government under contract number DE-AC05-96OR22464. Accordingly, the U.S. Government retains a nonexclusive, royalty-free license to publish or reproduce the published form of this contribution, or allow others to do so. for U.S. Government purposes.

\section{DISTABETTON OF THIS DOCUAENT IS UNLATTED.}

- Managed by Lockheed Martin Energy Research Corp. for the U.S. Department of Energy under contract number DE-AC0596OR22464. 


\section{DISCLAIMIER}

Portions of this document may be illegible in electronic image products. Images are produced from the best available original document. 


\section{DISCLAIMER}

This report was prepared as an account of work sponsored by an agency of the United States Government. Neither the United States Government nor any agency thereof, nor any of their employees, make any warranty, express or implied, or assumes any legal liability or responsibility for the accuracy, completeness, or usefulness of any information, apparatus, product, or process disclosed, or represents that its use would not infringe privately owned rights. Reference herein to any specific commercial product, process, or service by trade name, trademark, manufacturer, or otherwise does not necessarily constitute or imply its endorsement, recommendation, or favoring by the United States Government or any agency thereof. The views and opinions of authors expressed herein do not necessarily state or reflect those of the United States Government or any agency thereof. 


\title{
Potential Benefits of Using Commercial Simulators To Test Equipment Control Systems
}

\author{
Francis Ruppel and Wes Wysor \\ Oak Ridge National Laboratory" \\ P.O. Box 2008 \\ Oak Ridge, TN $37831-6003$
}

\begin{abstract}
Motivation is given for a technique to more thoroughly test semiconductor equipment control systems. A description is given of a simulator-based control system testing technique. Potential benefits that could be realized by using this technique in the semiconductor industry as well as benefits documented by using this technique in other industries are described. Specific requirements for using the technique in the semiconductor industry are outlined. A summary of a survey of nine commercial simulation systems is given. Finally, the outcome of the survey is compared with the requirements for using the technique.
\end{abstract}

\section{INTRODUCTION}

Studies at SEMATECH have shown that control system software failures account for some $40 \%$ of the equipment failures in a typical semiconductor fab [1]. The 1994 National Technology Roadmap for Semiconductors states that "Known reliability improvement processes need deployment throughout the industry for equipment reliability to achieve desired levels" [2]. Simulator-based control system testing methodology has proven effective in the aerospace, automotive, and other industries. This technique consists of developing a dynamic model of the semiconductor manufacturing equipment, loading the model into a simulator system, connecting the simulator to the control system, and testing the control system on the simulator rather than on the actual equipment. Fig. 1 shows an example of a simulator-based control system testing technique.

\section{POTENTIAL BENEFITS}

Control system testing using simulators may detect up to $30 \%$ of the control system software failures in the semiconductor manufacturing industry before the failures occur. Without a simulator, when a manufacturing tool is being designed and built the control system developer has to compete with other engineering and craft disciplines for access to the tool to develop and test the control system. Using a simulator, the control system developer can work in parallel with the other disciplines because full access to the simulator is available. This removes control system development from the project critical path. relieving the developer from equipment scheduling pressures.

A test using a simulator can be more comprehensive than a test using the actual tool and can also be automated. Using a simulator. the normal safety or process operational limits are not a concern, so the virtual test can transcend those limits. if necessary, to provide a more robust test. Less is spent on test consumables such as process gases, utilities, and test wafers because the virtual test uses none of these.

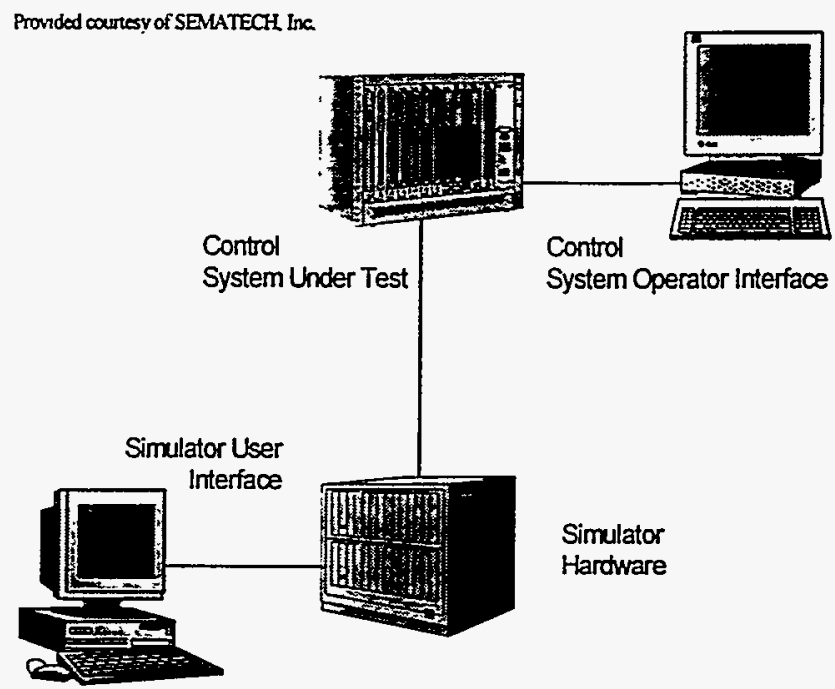

Fig. 1. Overview of Simulator-Based Control System Testing Technique.

It will always be necessary to test the control system on the actual tool because there are a small number of software bugs that cannot be detected using simulators. However, it is still advisable to have the simulator present during the test with the actual tool. During any lulls in activity, bugs that have been uncovered can be verified, changed, and tested using the simulator. This can save considerable time, especially if the bug does not occur until well into the process sequence, because simulated time can be advanced to reach the point in the process sequence where the bug occurs.

Quicker time to market has been documented in other industries. A manufacturer of anti-lock brake systems reported a three-to-one improvement when testing control systems using simulators first rather than testing such systems totally on automobile test tracks.

Finding and resolving a problem during design or testing is much quicker. and therefore cheaper. than when the problem is found after the equipment is operating in a fab. Reports from other industries claim that a problem can be corrected up to 20 times faster by using a simulator rather than trying to correct it in the field [3]. 
A semiconductor equipment supplier sells many different configurations of many models of its tool. Occasionally a problem may develop at an end-user site for which the supplier does not have a tool on-site with a similar configuration. Using a simulator, the supplier can mock up the system to uncover problems that would otherwise require troubleshooting in the field.

For semiconductor manufacturers, a simulator can be used to test new revisions of control system software. Many times a new revision introduces new bugs. The end-user must maintain two versions of software: a stable, old version and the unstable, new version. With a simulator, the new version can be extensively tested on the simulator until the confidence level is high enough to load the new version on the operating tool.

Finally, a simulator can be used to train operators using the actual tool control system and user-interface platform. The operator will gain experience with the dynamics of the process by using the simulator and will become familiar with the operation of the tool control system.

\section{EFFORT REQUIRED}

For semiconductor equipment suppliers who use this technique, the potential return on investment is high. How much effort is required to obtain a model suitable for control system testing? In Fig. 2 we propose that the best return on investment for activities related to simulator-based control system testing (software development, debugging, and new-release testing) and operator training is provided by a combination of input/output (I/O) loop-back tests and empirical modeling. Limited economic payback is provided by first principles and finite element modeling, which are oriented more towards concurrent engineering practices.

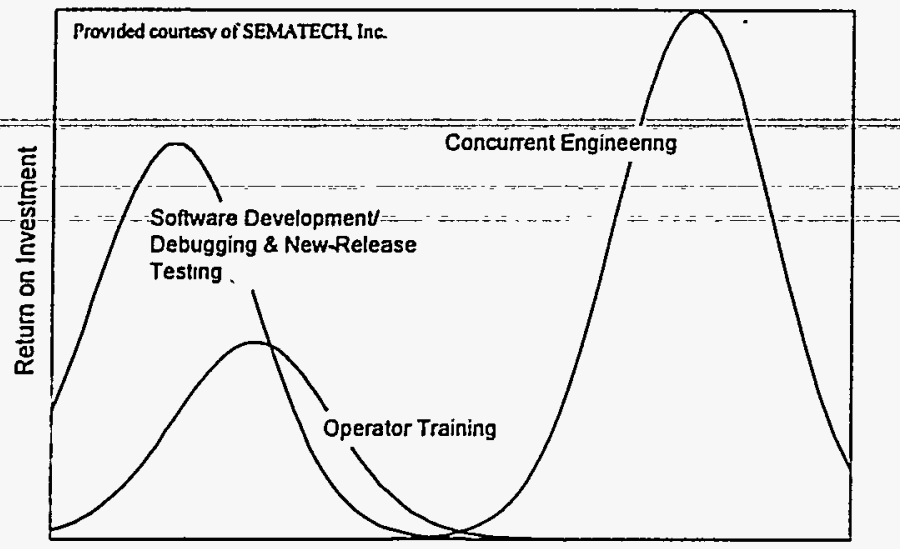

-1/O Loop-Backs- Emoincal Modeling- - First Pnnaples- Firmte ElementModeling Effort

Fig. 2. Proposed Return on Investment for Simulation-Related Activities.

An I/O loop-back test is a means to model a system at a very basic level when it involves discrete $\mathrm{I} / \mathrm{O}$ to the equipment.
Discrete outputs from the control system are matched with related discrete inputs to the control system, and the two are connected. For example, a control system may have an output that commands a gas valve to open. The gas valve may be equipped with a limit switch that provides an input to the control system when the valve is opened. In a simple I/O loopback test, the output signal, intended to operate the valve, would be rerouted to the input, intended to receive the limit switch status from the valve. A test of the control system software would exercise the valve output point and read the corresponding, simulated input point. Although this illustration describes a modification of the wiring harness, the same effect can be obtained by intercepting these signals at the bus level to simplify the simulator interface.

Empirical modeling techniques derive models from operating data. For dynamic systems, a technique known to control engineers as system identification is used. In this method, the output from the control system and the input back to the control system are continuously monitored. The control system output is made to perturb the system, and the resulting system response is monitored. By knowing the input to the system and the system's response, a mathematical. dynamic output-toinput relationship can be derived for the system. This relationship can be used to simulate the system for control system testing. This technique can actually provide a higher fidelity model than techniques requiring more effort, as shown in Fig. 2. Many of the simulation systems surveyed in this work have built-in tools to develop empirical models based on system identification experiments.

For operator training exercises, it is necessary to use more dynamic, empirical modeling to develop the simulator model to provide the operator with more realistic system behavior.

It would be advantageous for semiconductor equipment suppliers to leverage the capabilities of commercial simulators. However, the simulator requirements that are unique to the semiconductor industry must be addressed before it is beneficial for a semiconductor equipment supplier to invest in offthe-shelf simulators used in other industries.

\section{APPROACH}

Oak Ridge National Laboratory has worked with SEMATECH to present guidelines for simulator-based control system testing. Part of the work was a documentation of requirements gathered from semiconductor equipment suppliers and a survey and characterization of nine commercially available simulator systems. The culmination of the work is a report issued by SEMATECH [4], which is available by following the links to technical reports on SEMATECH's Web site at http://www.sematech.org.

\section{REQUIREMENTS}

Requirements specific to the semiconductor industry for using the simulator-based testing method were documented. 
The requirements were assembled from meetings during the first half of 1996 with representatives of Applied Materials. Lam Research, Novellus, Ultratech Stepper, Watkins-Johnson. and the SEMATECH working group for the project. The working group included members from AMD, IBM, National Semiconductor, Motorola. and Texas Instruments. Some requirements resulted from discussions with personnel at Semitool before the beginning of the formal project. The requirements are summarized as follows.

\section{Interfacing Capability}

Typical cluster tools have more than 1000 analog and digital I/O lines and many serial interfaces. The effort involved in wiring to each point would be overwhelming. For a simulator-based tester to be useful to major semiconductor equipment suppliers, an interface between the simulator and control system needs to be implemented that is higher in the system architecture than the physical I/O level. Software I/O drivers can be configured for two modes: normal and test. In test mode, commands are rerouted from the actual, physical I/O hardware to an address in the simulator.

Electrical interfacing between a tool controller I/O hardware and the hardware available from a commercial, off-theshelf simulator is not standardized. Time and effort are required to match the signal levels between the two systems. This lack of standardization provides an additional incentive to interface at a higher level in the system architecture.

Controllers for semiconductor manufacturing equipment interface to many serial devices. The fraction of $\mathrm{I} / \mathrm{O}$ interfaces using serial and smart $\mathrm{I} / \mathrm{O}$ devices is larger for semiconductor manufacturing equipment than for other industries. Commercial simulators do not currently provide the necessary number and types of serial interfaces required. Simulators should be provided with more serial interface options.

\section{System Configuration}

Given the number of product groups and configurations available from semiconductor equipment suppliers, in some cases more than 100 different system configurations exist. Many times, further customization is required beyond the standard configurations. A simulator should be able to be reconfigured easily so that a single simulator system can be used to test more than one product model.

\section{Support for Automated Testing}

Semiconductor equipment suppliers have said that up to $75 \%$ of their control system software deals with exception handling. The simulation system should be capable of injecting perturbations and fault conditions on a deterministic or random basis. Simulated response times of hardware devices should be allowed to vary from instantaneous to a few times slower than the actual device.
Simulators should also support unattended-mode testing. Simulator-based automated testing and report generation would greatly help semiconductor equipment suppliers meet schedule demands while increasing the reliability of their delivered equipment. Semiconductor equipment suppliers cannot afford to dedicate millions of dollars of product equipment for extended controller software testing. The simulator should be capable of recording parameters, events, and test data.

\section{Cost}

Major semiconductor equipment suppliers sell many units at prices of about one million dollars. Compare this with a major chemical plant that could cost hundreds of millions of dollars or with an advanced military aircraft that could cost a billion dollars. In these cases, a million-dollar simulation system represents a small fraction of the total system cost. For a simulator to be cost-effective to a semiconductor equipment supplier, the cost of the simulation system (including model development) should be less than the semiconductor equipment supplier's cost for the actual processing equipment. Otherwise, after the cost of labor, clean room space, and consumables is accounted, it may be more economical for the supplier to set up dedicated processing equipment on which control system testing could be performed. Hence, some equipment suppliers believe that in this industry, cheaper, generalpurpose, less comprehensive simulation systems have a better return on investment than expensive, customized, more comprehensive simulation systems. However, the bottom line in cost is that the life-cycle return on investment should be high. Since every case has different economic driving forces, a strict purchasing cost requirement cannot be specified.

\section{Simulation Capability}

Semiconductor equipment suppliers do not normally test the dynamics of off-the-shelf devices, such as servo-controlled positioning stages that plug into their control system communications bus. It is acceptable to treat these subsystems as "black boxes." Furthermore, it has been claimed that 60 to $90 \%$ of the capability of control software can be tested without exercising the $\mathrm{I} / \mathrm{O}$ at the hardware level. Most of the software is not related to the critical timing or control actions taken by the control system. Equipment suppliers have expressed a need for more support for event-oriented simulation in addition to simulation tools for detailed. continuous equipment dynamic behavior. Material and recipe flow need to be tested, and a simulation should be capable of sequencing the virtual movement of wafers between chambers of a cluster tool.

A key feature that would benefit the industry would be to provide building blocks or a library of standard components for equipment simulations. For example, a standard dynamic model of a mass-flow controller that could be "plugged" into a simulator would be used by a majority of semiconductor equipment suppliers. 


\section{Ease of Use}

The growth rate of the semiconductor industry is high, which means that new employees are being hired at a high rate. Even without the high growth rate, the semiconductor industry is highly dynamic in that professionals move from one company to another more frequently than in other industries. Therefore, the simulator needs to have the capability of being self-taught by new employees within one or two weeks . The simulator should have many online help and tutorial utilities. A graphical user interface with intuitive, object-oriented features is preferred, and it should follow a consistent and acceptable style for color usage and information display. Minimal steps should be required to build models and displays.

\section{Services}

Off-the-shelf solutions are encouraged as much as possible, but semiconductor equipment suppliers are also interested in turnkey solutions, whereby they could contract the task of developing a simulator, including the development of a baseline model for their equipment.

\section{SURVEY}

Given the requirements from the previous section, a survey of nine commercial simulator systems was performed to see if overlap exists between semiconductor industry requirements and off-the-shelf capability. The selection criteria for the systems chosen for the survey were as follows: (1) a platform comprising real-time simulator hardware and/or associated simulation software that can (2) interface to open architecture, real-time control systems prevalent in the semiconductor industry (e.g., VME-bus, STD-bus, PC/104, and PC-based) and that can (3) run simulations applicable to semiconductor manufacturing equipment for the purpose of testing the equipment control system. Although other commercial products may meet these criteria, Table 1 shows the products only from companies who agreed to participate in the survey. In the survey, the simulator systems were functionally characterized for the requirements outlined previously.
Table 1. Products Surveyed.

\begin{tabular}{|l|l|}
\hline \multicolumn{1}{|c|}{ Company } & \multicolumn{1}{c|}{ Product } \\
\hline $\begin{array}{l}\text { Applied Dynamics Inter- } \\
\text { national }\end{array}$ & SIMsystem \\
\hline dSPACE & dSPACE \\
\hline EAI Simulation Associates & ARTS \\
\hline Integrated Systems, Inc. & MATRIX $/$ RealSim \\
\hline The MathWorks & MATLAB/SIMULINK \\
\hline MGA Software & ACSL/ACSLrt \\
\hline National Instruments & LabVIEW \\
\hline S-S Technologies, Inc. & $\begin{array}{l}\text { PICS Simulation for } \\
\text { Windows }\end{array}$ \\
\hline Visual Solutions & VisSim/RT \\
\hline
\end{tabular}

Detailed results for each characterization are given in the referenced SEMATECH report. In general, the results of the survey show that some support already exists for high-level interfacing between simulators and control systems. Some suppliers provide the capability already; some provide tools that only enable high-level interfacing; and some provide only conventional analog and digital interfacing options.

Advanced tools are available to help the semiconductor equipment supplier manage simulations of many different equipment models. High-level development tools enable models to be built with minimal work.

The requirements for automated testing are almost completely met by commercial simulators.

System costs reviewed in this work range from less than $\$ 10.000$ to more than $\$ 100,000$. Typical costs of model development or system integration were not surveyed, but with the wide range of purchasing costs found, it is likely that a high return on investment can be realized by using a commercial simulation solution.

Event-oriented simulation capability is apparently just becoming available from simulator suppliers. A few suppliers have the capability already, and many more will have it soon. (One supplier released a new software version with this capability subsequent to the publication of the SEMATECH report.) For continuous modeling, simulator suppliers offer little support for special modeling blocks for semiconductor processes at present, but control analysis tools are readily available.

For ease of use, about half of the simulation systems offer software with advanced tools such as context-sensitive help and online tutorials.

Services are available from most simulator suppliers or from associated third parties to develop models and perform systems integration. 


\section{CONCLUSION}

Semiconductor equipment suppliers can realize many benefits from employing simulator-based control system testing. In particular, semiconductor equipment reliability will increase if equipment suppliers use simulators to test their control systems before equipment delivery. Although there is overlap between requirements for simulators for control software testing in the semiconductor industry and the features of commercially available simulators, there are other -require-ments-that-are-not-fully-matched-at-this-time-Semiconductor equipment suppliers are encouraged to pursue areas of overlap between their requirements and the simulator system vendor's capabilities. Simulator system vendors are encouraged to extend -their-capabilities-to areas-where-requirements-have-no matching capabilities.

\section{REFERENCES}

[1] H. Wohlwend, "Software Reliability in the Semiconductor Industry," Advanced Process \& Equipment Control Workshop VIII." Santa Fe, N.M., October 27-30, 1996.

[2] "The National Technology Roadmap for Semiconductors," Semiconductor Industry Association, San Jose, Calif., 1994.

[3] K. Pritchard, "Applying Simulation to the Control Industry," Control Engineering, Cahners Publishing Co., May 1989.

[4] F. Ruppel and W. Wysor, "Guidelines for Simulator-Based Control System Testing, ${ }^{21}$-97023237A-TR, SEMATECH, Austin -Tex,-May 31,-1997. 